\title{
Multicompany trials adapt to disciplines beyond cancer
}

When the I-SPY 2 trial launched in 2010, oncologists heralded it as the future of cancer research. Five pharmaceutical companies put aside their differences to participate in the landmark phase 2 breast cancer trial, which adaptively and efficiently randomized patients to one of seven experimental therapies. Now, even as I-SPY 2 propels its first two drugs into phase 3 trials, researchers in other areas of medicine are catching on to the benefits of this collaborative approach. On 11 December, Europe's Innovative Medicines Initiative (IMI) announced a €53 million ( $\$ 73$ million) call for proposals for a similarly designed trial in Alzheimer's disease. Already, at least 12 drug companies are keen to participate.

"I think this is a step in a right direction to really try to beat Alzheimer's in a serious way," says Randall Bateman, a neurologist at the Washington University School of Medicine in St. Louis. Bateman is coordinating an ongoing collaborative and adaptive trial called DIAN-TU, which is testing two experimental antibody drugs from Eli Lilly and Roche in patients with dominantly inherited forms of early-onset Alzheimer's disease. The bigger IMI-initiated trial, he says, is likely to offer hope to a much larger patient population.

The Brussels-based IMI is seeking to launch a phase 2 trial that will break new ground by using biomarker data and preliminary efficacy results to randomize patients to the most promising of various treatment arms using an adaptive prepare their proposals for the IMI publicapproach. Researchers have until 8 April to

private partnership, which hopes to have the project underway by the end of the year.

Would-be IMI investigators, however, face several key design dilemmas. I-SPY 2 succeeded in part by making the most of a relatively defined patient population with a fast-progressing disease, and it benefited from informative biomarkers and accepted endpoints that can guide adaptive randomization. Those joining the IMI trial, by contrast, will have to deal with a less defined population of 'at-risk' individuals with a slow-progressing disease, with only experimental biomarkers and unproven endpoints to guide the way.

That said, traditional Alzheimer's trials have these same problems, without the benefits of the adaptive and collaborative elements. And some of the efficiencies come easy. If five companies each run a two-armed trial to test their lead Alzheimer's candidate, they cumulatively have to enroll patients into ten trial arms. A single protocol that can test five drugs in only six arms provides an immediate savings of $40 \%$ in enrollment, patient-monitoring and follow-up costs. For Alzheimer's drug developers who have been repeatedly burned by costly failures in past years, that's money in the bank.

The Lung Cancer Master Protocol, spearheaded last year by the Washington, DCbased think tank Friends of Cancer Research, showcases another advantage of collaborative studies. In their planned phase $2 / 3$ trial, which won't incorporate adaptive randomization, investigators will assign patients to appropriate molecularly determined treatments on the basis

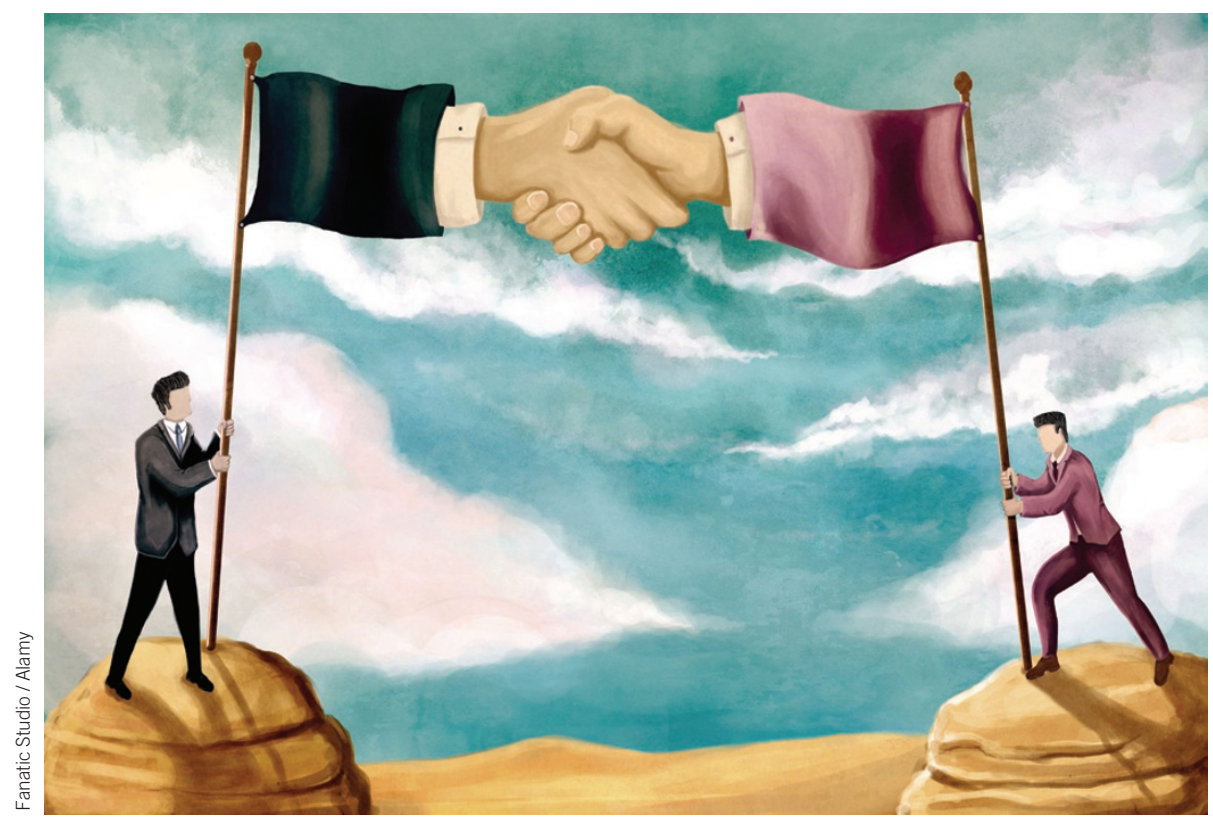

of a single biomarker screen. This setup lowers the biopsy burden and boosts enrollment efficiency. The trial is set to start testing five drugs from four different sponsors in March and can be expanded to include more drugs and sponsors.

\section{I spy more opportunities}

Drug companies, who are typically loath to relinquish control of their experimental agents, would have spurned these types of trials a decade ago. But as high failure rates have continued to take their financial toll, competitors are now embracing collaborative efforts of all varieties. With I-SPY 2, DIAN-TU and a few other precedents blazing a path, specialists in other indications are starting to follow.

Melissa Paolini, director of advanced trials at QuantumLeap Healthcare Collaborative, a California-based partnership between academic scientists and biotech entrepreneurs that is sponsoring the I-SPY 2 trial, says the cooperative platform works for any high-risk patient population in which early or surrogate markers of response can be used to accelerate decisions. "And that is a very large boat," she says. Most of her discussions are still in the cancer field, but she is also in preliminary conversations with a group interested in studying new therapeutics for amyotrophic lateral sclerosis.

IMI scientific manager Elisabetta Vaudano, an architect of the recent plan for the adaptive 'master protocol' to test Alzheimer's therapeutics, sees interest in other disease areas, too. "Diabetes companies are starting to put their heads together and say this could be a good idea," she says. "It could happen in six months' time."

Meanwhile, Donald Berry, a biostatistician at the University of Texas MD Anderson Cancer Center in Houston and a principal investigator for I-SPY 2, is working with an international group of academic scientists to launch a similar trial for the treatment of severe acute respiratory infections. As envisioned, this trial would test multiple therapies, all while adapting enrollment according to factors such as geographical variability in responses.

Drug developers working on anti-infective agents, autoimmune diseases, Parkinson's and a host of cancer indications have also spoken to Berry, who runs a statistical consulting group with his son that specializes in adaptive trials, about launching similar trials. (The Austin, Texas-based consultancy is already collaborating on a proposal for the IMI Alzheimer's study.) "I think this approach is useful for everything," Berry says.

Asher Mullard

Across the divide: Drug companies are teaming up to run trials in many disease areas. 